\title{
Progressive vitiligo, mental retardation, facial dysmorphism, and urethral duplication without chromosomal breakage or immunodeficiency
}

\author{
Philippe Labrune, Rémy Assathiany, Dominique Penso, Michel Odièvre
}

\begin{abstract}
A boy, born to first cousin parents of Algerian origin, first presented at the age of 9 years with growth failure, mental retardation, and dysmorphic facies. Progressive vitiligo developed from the age of 12 and distal duplication of the urethra was later recognised. The basis of this syndrome remains to be determined; autoimmune disease, chromosomal breakage syndromes, and other neurocutaneous syndromes have been excluded.
\end{abstract}

Neurocutaneous syndromes are a well classified group of disorders, and new entities are described regularly. We report here a boy who presented with retarded growth, moderate microcephaly, mental retardation, progressive vitiligo, and urethral duplication. Neither immunodeficiency nor chromosomal breakage could be detected. Thus, this clinical association may represent a new syndrome.

\section{Case report}

This boy is the fourth child of first cousin Algerian parents whose first three children were normal. He was born in Algeria and his weight and length were said to be normal at birth. However, his psychomotor development was considered to be slower than it had been in his three sibs. The child was first admitted to our unit at the age of 9 years. His height was $115 \mathrm{~cm}$ ( $-3 \mathrm{SD}$ ), weight was $18.5 \mathrm{~kg}$ ( $-2 \mathrm{SD})$, and head circumference was $50 \mathrm{~cm}(-2 \mathrm{SD})$.
Mild facial dysmorphism was noted with a beaked nose and a high, narrow palate. Moderately severe mental retardation was also present (IQ 65 on the Stanford-Binet scale). Complete blood cell count, serum and urine electrolytes, and liver tests were normal, as were serum and urine amino acid chromatography. The karyotype (enzymatic denaturation, 15 metaphases studied) did not show any cytogenetic abnormality. At the age of 12 vitiligo was first noted.

The patient was readmitted at the age of 15 years. His height was $140 \mathrm{~cm}(-3 \mathrm{SD})$, weight was $29 \mathrm{~kg}(-2.5 \mathrm{SD})$, and head circumference was $50 \mathrm{~cm}(-3 \mathrm{SD})$. The facial dysmorphism (fig 1) and mental retardation were still present. Disseminated vitiligo was also noted (fig 2). Physical examination showed moderate macrogenitosomia and two separate urethral meatuses, while pubertal development was progressing. Ophthalmological examination proved normal, as did total skeletal radiographic examination. Antinuclear factors, anti-DNA autoantibodies, and Waaler-Rose latex test were negative in serum. Complement components were normal and the search for numerous serum autoantibodies was negative (anti-smooth muscle, anti-mitochondria, antithyroid, anti-gastric, anti-insulin, and antiislet cells). Thyroid hormones, ACTH, and cortisol plasma concentrations were normal. A second karyotype was performed (cultured with trimethoprim, 58 metaphases studied) and no evidence of fragile $\mathrm{X}$ was found. At the age of 17 , his height was $155 \mathrm{~cm}$, his weight was $35 \mathrm{~kg}$, and his head circumference was $51 \mathrm{~cm}$. Vitiligo had progressed and the skin
Service de Pédiatrie, Hôpital Antoine Béclère, 157 rue de la Porte de Trivaux, 92141 Clamart Cedex, France.

P Labrune

R Assathiany

D Penso

M Odièvre

Correspondence to Dr Labrune.

Received 30 July 1991. Revised version accepted 2 January 1992

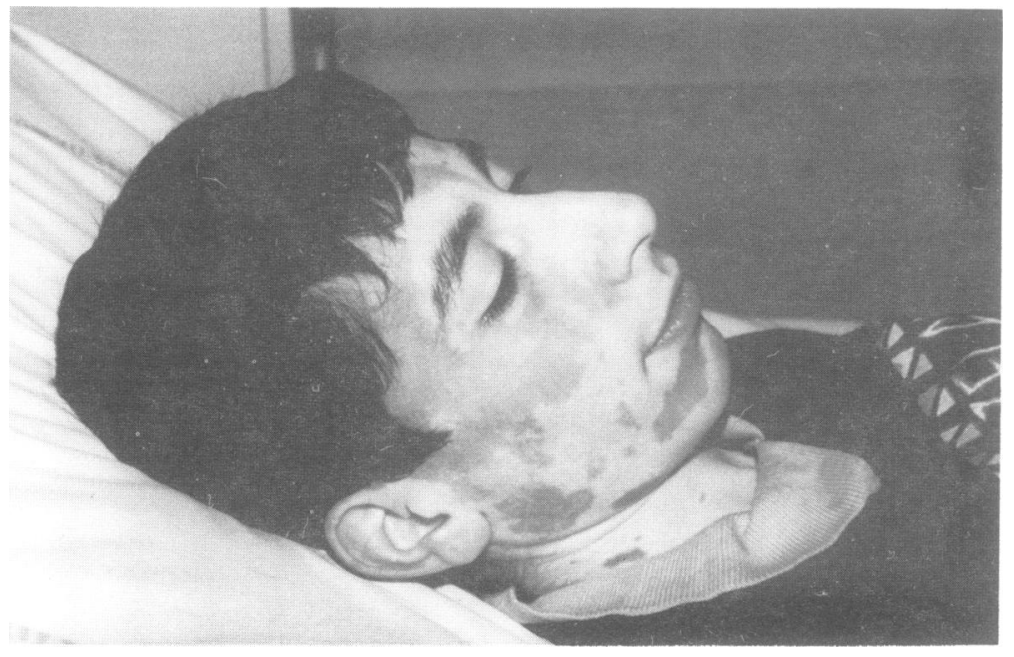

Figure 1 Facial profile of the child showing the mild dysmorphism (beaked nose) and the vitiligo. 

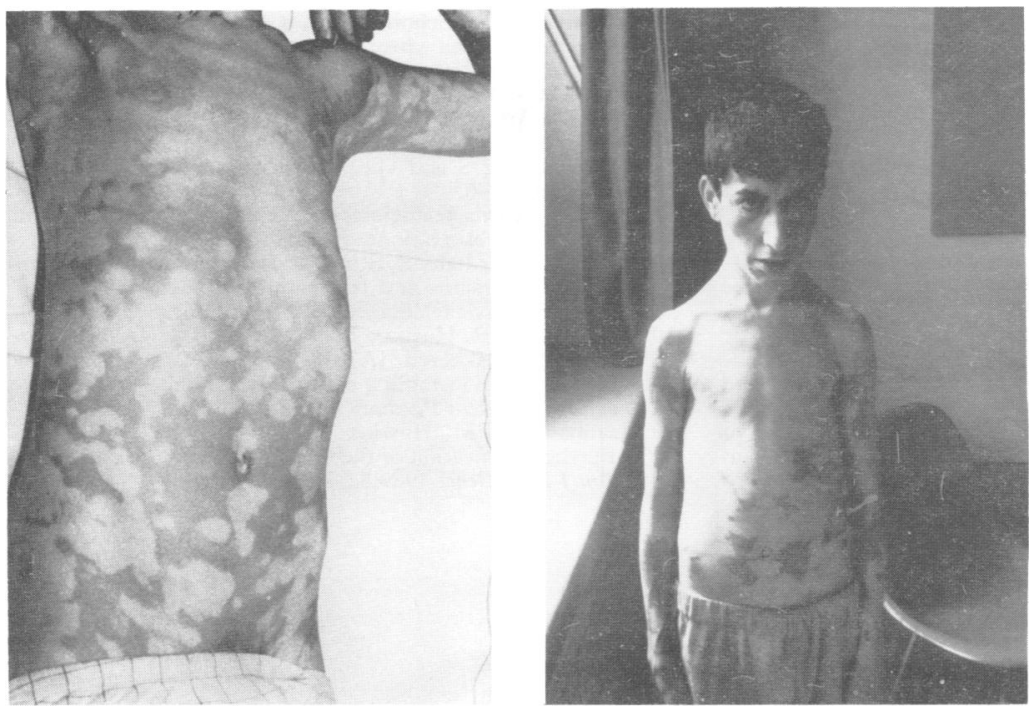
Figure 2 (Left) vitiligo at the age of 15, (right) increase of the vitiligo at the age of breakage inducing agents ( $R$ banding after stimulation with phytohaemagglutinin and IL2): no chromosomal breakage was observed in 50 mitoses ( $\mathrm{Dr}$ Aurias, Institut Curie, Paris).

\section{Discussion}

Our patient presented with retarded growth, mental retardation, progressive vitiligo, and urethral duplication, with normal immunological and cytogenetic studies. The close parental consanguinity suggests that this disorder is transmitted as an autosomal recessive trait.

Vitiligo is known to be a marker for certain autoimmune diseases, such as thyroid or Addison's disease. These disorders were excluded in our patient since plasma hormone concentrations were normal and no autoantibodies were found. Vitiligo has also been reported in syndromes such as the Ermine phenotype, ${ }^{1}$ but our patient had no evidence of hearing loss.

Several possibly related neurocutaneous syndromes have been previously described. Lison $e t a l^{2}$ and Mukamel $e t a l^{\beta}$ reported two families with consanguineous parents, in whom several sibs presented with spastic paraparesis, muscle wasting, mental retardation, skeletal deformities, and cutaneous manifestations (greying hair and hypopigmented and hyperpigmented lesions). A syndrome of spastic paraplegia, cutaneous lesions, and dysarthria was reported by Bahemuka and Brown, ${ }^{4}$ but the skin rash was confined to the face. Our patient had no evidence of paraparesis, no skeletal deformities, and his cutaneous lesions were different from those reported in these studies. ${ }^{2-4}$

In our patient, the absence of chromosomal and immunological defects excludes chromosomal breakage syndromes such as Fanconi's anaemia, Bloom's syndrome, ${ }^{56}$ xeroderma pigmentosum, and ataxia telangiectasia. ${ }^{78}$

Finally, the diagnosis of Nijmegen breakage syndrome was suspected since it includes most of the clinical manifestations of this patient. ${ }^{910}$ However, the lack of serious infections and of chromosomal abnormality strongly argues against this diagnosis.

Therefore, our patient seems to have a syndrome different from previously reported neurocutaneous syndromes and from the chromosomal breakage syndromes.

Immunological studies.

Serum immunoglobulins $(g / \mathbf{l})$

IgG: 16

IgA: 1.35

accinal antibodies

Antipoliomyelitis type $1: 1 / 640$

Antipoliomyelitis type $2: 1 / 1280$

Antipoliomyelitis type $2: 1 / 1280$

Antipoliomyelitis type $3:$

Aymphocyte populations (\%)

Lymphocyte 72

T3: 72

T8: 12

Mitogen and antige

PHA: $203(\mathrm{~N}>15)$

Tuberculin: $24(\mathrm{~N}>5)$

Candidin: $31(\mathrm{~N}>5)$

Tetanus toxoid: $16(\mathrm{~N}>5)$
Figure 3 Moderate macrogenitosomia with two visible urethral meatuses. 
We are grateful to Dr Aurias for performing the search for chromosomal breakage.

1 O'Doherty NJ, Gorlin RJ. The Ermine phenotype: pigmentary-hearing loss heterogeneity. Am $\mathcal{f}$ Med Genet 1988;30:945-52.

2 Lison M, Kornbrut B, Feinstein A, et al. Progressive spastic paraparesis, vitiligo, premature graying and distinct facial appearance: a new genetic syndrome in three siblings. Am ₹ Med Genet 1981;72:557-64.

3 Mukamel M, Weitz R, Metzker A, Varsano I. Spastic paraparesis, mental retardation, and cutaneous pigmentation disorder. A new syndrome. Am $₹$ Dis Child 1985;139:1090-2

4 Bahemuka $M$, Brown JD. Heredofamilial syndrome of spastic paraplegia, dysarthria and cutaneous lesions in spastic paraplegia, dysarthria and cutaneous lesion
five siblings. Dev Med Child Neurol 1982;24:519-24.
5 Chaganti RSK, Schonberg S, German J. A many-fold increase in sister chromatid exchanges in Bloom's synincrease in sister chromatid exchanges in Bloom's syndrome lymphocyte

6 Aurias A, Antoine JL, Assathiany R, Odièvre M, Dutrillaux $B$. Radiation sensitivity of Bloom's syndrome lymphocytes during $\mathrm{S}$ and $\mathrm{G} 2$ phases. Cancer Genet Cytogenet 1985;16:131-6.

7 Boder E. Ataxia telangiectasia: some historic, clinical an pathologic observations. Birth Defects 1975;XI:255-70.

8 Aurias A, Dutrillaux B, Buriot B, Lejeune J. High frequencies of inversions and translocations of chromosomes 7 and 14 in ataxia telangiectasia. Mutat Res 1980;69:369-74.

9 Weemaes CMR, Hustinx TWJ, Scheres JMJC, Van Munster PJJ, Bakkeren JAJM, Taalman RDFM. A new chromosomal instability disorder: the Nijmegen breakage syndrome. Acta Paediatr Scand 1981;70:557-64.

10 Taalman RDFM, Hustinx TWJ, Weemaes CMR, et al. Further delineation of the Nijmegen breakage syndrome. Am 7 Med Genet 1989;32:425-31. 